\title{
Multiobjective Metaheuristic Load Control Algorithm for Interaction between Smart Home and Humans
}

\author{
Hao Chi $\mathbb{i D}^{1,2}$ and Sang-ug Kang $\mathbb{D}^{1}$ \\ ${ }^{1}$ Department of Computer Science, Sangmyung University, Seoul 03016, Republic of Korea \\ ${ }^{2}$ Organization Propaganda Department, Shandong Vocational and Technical University of International Studies, \\ Rizhao 276800, China \\ Correspondence should be addressed to Sang-ug Kang; 2011020116@st.btbu.edu.cn
}

Received 4 December 2021; Revised 24 December 2021; Accepted 8 January 2022; Published 8 February 2022

Academic Editor: Vijay Kumar

Copyright $(2022$ Hao Chi and Sang-ug Kang. This is an open access article distributed under the Creative Commons Attribution License, which permits unrestricted use, distribution, and reproduction in any medium, provided the original work is properly cited.

\begin{abstract}
Technology has boosted electric power consumption both locally and worldwide, resulting in a substantial increase in demand for electric power. A multiobjective smart house human-computer interaction load control method is suggested to meet the goal of lowering power usage and pricing in smart home load control. It presents a model that includes marginal costs and establishes an electricity price model that takes the load rate into account. It identifies switch appliances and temperature control appliances and gathers human activities, indoor and outdoor temperature, and light intensity using intelligent equipment to create a multiparameter comfort model. It creates a multiobjective model of comfort and electricity price with the purpose of reducing electricity price and multiparameter comfort, and it improves particle swarm performance by using a distance ratio based on fitness value. The optimization algorithm solves the model, determines the best smart home human-computer interaction load control scheme, creates the smart home remote control system's functional modules, and optimizes the multiobjective smart home human-computer interaction load control algorithm using a frequency-duration parameter tracking learning model. The testing findings suggest that the proposed algorithm can cut power prices in a reasonable manner, as well as regulating $40 \%$ reduced electricity usage and load in smart homes.
\end{abstract}

\section{Introduction}

The use of Internet of Things technology to link various types of home equipment in order to give users a combined control solution for home equipment in various modes is known as smart house $[1,2]$. A smart house is a home with Internet-connected gadgets that allow for control of appliances and distant monitoring and consider systems like heating and cooling. Human-computer interaction technology predicts that home life facilities will evolve into a high-level family living environment suited for humans to dwell in $[3,4]$. If some AI-enabled and metaheuristic approaches are applied in smart homes, they can not only increase the quality of life but also save money $[5,6]$. Touch screen control, video control, and voice control are the most common methods of human-computer interface in smart homes [7]. To realise the human-computer interaction function, these control methods need to control different home equipment, which generates different electrical loads. In the long-term control process, the rising peak load of electricity generated leads to the smart home's environmental load experiencing excessive regulatory pressure $[8,9]$. In the field of smart home, researchers are constantly making new research at the technical level [10]. However, merely addressing the hardware environment and not addressing the software environment when designing a comprehensive smart home system would overlook the user's unique experience as well as the user's internal demands $[11,12]$. To realise the goal of harmonious and individualised human-computer interaction, a set of 
intelligent multiobjective smart home human-computer interaction load control support is required in addition to these intelligent hardware and software capabilities $[13,14]$.

People's reliance on energy is at an all-time high these days, as power consumption has risen steadily in recent years. In both residential and commercial buildings, it is important to consider monitoring and evaluating the electricity system or devices that are used on a daily basis. The energy system becomes imbalanced when there is inadequate power plant capacity to meet demand (load) from all users, which can result in a nationwide blackout that can take days to recover from. We have focused on the topic of electricity shortages in this study and developed a strategy to assist minimise energy use in the home, office, or any other desirable location.

Highlights of the present study are as follows:

The paper proposes a metaheuristic approach for multiobjective smart home human-computer interaction load control algorithm. It is challenging to handle the load without considering multiple parameters and in order to consider multiple parameters, there is a need to devise a multiobjective metaheuristic approach which can consider multiple criteria for optimal consumption of electricity by balancing the load of energy consumption.

For experimental purpose, a house is taken as the research object having area of 109 square meters. It integrates the full domestic smart home equipment, including smart lighting systems and smart electrical control, using integrated wiring technology, security technology, automated control technology, network communication technology, and audio and video technology.

The results obtained are projecting that the proposed work optimises the consumption of customer electricity, power load fluctuations, convergence rate, and load control by using metaheuristic approach in smart homes.

The rest of the paper is organised as follows: Section 2 describes the state of the art in the field of IoT to design smart homes for electricity consumption reduction. In Section 3, we describe the methodology and multiparameter comfort model construction. In Section 4, we elaborated optimal design of multiobjective smart home human-computer interaction load control algorithm and in Section 5 we discussed the results followed by conclusion.

\section{Related Works}

Relevant research on smart home human-computer interaction load control has achieved preliminary results at this stage. In [15], the authors have proposed the applicability of hybrid broadcast broadband TV to a unified smart home experience. Hybrid broadband broadcast (HBB) TV is a standardised project designed to provide consumers with broadband and broadcast facility of transmission of services through the collaboration of connected TVs, STBs, and multiscreen devices. A smart home is a home facility in which home appliances and equipment may be operated automatically from any networked device. An architecture that uses the TV companion screen and multimedia synchronisation $\mathrm{HBB}$ framework is proposed to realise the seamless integration of the smart home environment and the handheld devices that support hybrid broadcast broadband TV and consumers (such as smart phones and tablets). Subjective tests based on average opinion scores are used to measure the benefits of the proposed system and quality of experience is taken into consideration. Users can have a satisfying experience and excellent perception of the merged results of TV and smart home services owing to the exact design of hybrid services delivered via hybrid broadcast broadband TV.

In [16], the authors have proposed the status quo and challenges of smart homes related to the context-aware Internet of Things. Smart homes are considered to be an important field of IoT applications. It is a system where a home is interconnected with various types of things interacting through the Internet, which helps make the home intelligent and interconnected. However, due to its remote control capability, it also brings great concerns to users' privacy and safety. Therefore, the rapid development of the Internet of Things technology has brought huge challenges for home users. To answer the concerns of how to provide safe and dependable services to home users, how to preserve account privacy, and how to successfully run smart homes in regulated settings to prevent personal data theft, further research is needed. Different methods are proposed to overcome these problems.

This research review examines the methodologies and challenges of smart homes, as well as prospective remedies and explanations of the issues that remain unsolved. In [17], the authors have proposed a smart home environment control man-machine interface based on asynchronous electrooculogram, aiming to provide daily assistance for patients with severe spinal cord injury (SCI). There are several buttons, each corresponding to a control command, flashing randomly on the graphical user interface. Each flash of the button serves as a visual cue for the user to blink. To issue a control command, the user can flash synchronously with the flashing of the corresponding button. The blink is identified based on the recorded EOG signal, and the target button and its related control instruction are determined. The suggested technique provides a simple and practical method for severely injured patients to control their smart home environment, which is beneficial to their everyday lives.

In [18], an integrated solar energy is deployed as an implanted system in a smart house. The control technique presented controlled both desegregated energy space and resources obtained from the structure power efficiently. This was done in order to keep the grid electricity price as low as possible in the outset, such as for individual households. The model designed a load dispatch curve which is used to produce the resultant load scheduling. 
Although the above methods have made some progress, yet the smart home human-computer interaction load control is not accurate. This research investigates a multiobjective smart home human-computer interface load control method to address the aforementioned issues. The smart home human-computer interaction load control is targeted at groups of users as well as a single user. The control of the home equipment is diversified, realizing efficient and economical control of smart home humancomputer interaction. Research shows that the designed human-computer interaction loads control algorithm can save the electricity cost and electricity consumption of smart homes. It also reduces the load so that the house achieves smart home human-computer interaction while consuming the least amount of power.

\section{Methodology}

\subsection{Multiobjective Smart Home Human-Computer Interaction Load Control Algorithm}

\subsubsection{Model Construction}

(1) Electricity Price Model Taking into Account Load Rate. In order to ensure fair burden and optimize the efficiency of resource allocation, analyse the marginal power supply cost of each electricity user, and determine the basic electricity price for each electricity user. In a smart home humancomputer interaction control system, the basic energy price normally recovers all marginal capacity costs, and the settlement is determined based on the electrical load condition at the time of the user's maximum load [19]. However, in the real world, operability and measurement circumstances must be taken into account, and the basic power price is usually calculated based on the maximum load [20]. If the power system and the user have high cross-peak power consumption, the user's real marginal capacity cost will be lower than the partial capacity cost, which will help to solve the concerns mentioned above.

According to international laws, users of various voltage levels are divided into levels 1 to 5 according to the load rate. Assuming that $v$ represents the voltage level of users in the $i$ gear, (1) represents the marginal transmission and transformation capacity $\operatorname{cost} B_{m r, i}$ :

$$
\left\{\begin{array}{l}
B_{m r, i}=d_{i} \times B_{r}^{v}, \\
B_{r}^{v}=B_{s, t}^{v}+B_{s, t}^{j-v}, \\
B_{s, t}^{j-v}=k_{j-i} \times B_{s, t}^{j} .
\end{array}\right.
$$

In (1), $d_{i}$ represents the set $i$ user system simultaneous rate; $B_{r}^{v}$ and $B_{s, t}^{v}$, respectively, represent the system marginal transmission and transformation capacity cost at voltage level $v$ and the average transmission and transformation investment cost of the current level of voltage $v$ during the calculation period. The values of $B_{s, t}^{v}$ and $B_{s, t}^{j}$ are calculated according to the voltage transmission and transformation investment annuity of each level. $k_{j-i}$ and $B_{s, t}^{j-v}$, respectively, represent the proportion of the power transmission from the adjacent voltage level $j$ to the adjacent voltage level $i$ and the transfer of the high voltage level $j$ to the voltage level $v$. The average investment cost of transmission and transformation.

According to (1), it can be seen that $d_{i} \times B_{r}^{v}$ represents the marginal capacity cost of the $i$ user; the electricity consumption $f_{i} * 1$ corresponding to the maximum load of the user unit is related to $\left(d_{i}-a_{i}\right) * B_{r}^{v}$. In order to basically recover the electricity price, the electricity consumption of $f_{i} \times 1$ is independent of $a_{i} \times B_{r}^{v}$. Among them, $a_{i}$ represents the power fluctuation coefficient.

After the above analysis, the marginal cost is used to complete the pricing, and the financial balance is used to implement adjustments. Equation (2) represents the user's basic electricity price $O$ :

$$
O=k\left(\frac{1}{1-s} \times o_{\text {buy }}+\frac{\left(d_{i}-a_{i}\right) \times B_{r}^{v}}{720 \times f_{i}}\right) .
$$

In (2), $k$ and $s$, respectively, represent the financial balance adjustment coefficient and the user's power-received voltage level grid loss rate; $o_{b u y}$ represents the average power purchase price of the grid company.

(2) Multiparameter Comfort Model. The planned smart switch collects data on human activity, light conditions, and interior and outside temperature, with normal temperature being utilised to represent both indoor and outdoor temperatures [21]. Divide the light situation $Z$ according to the 5 levels of light intensity, $Z=[1,2,3,4,5]$. The value is arranged from low to high, and the light intensity is proportional to the magnitude of the value. It indicates the human body activity based on whether or not there is a human being present.

It reflects the comfort of the human body for electrical appliances from two parts. One is the comfort of switches, such as lamps and televisions, and the other is the comfort of adjustable electrical equipment, such as water heaters and air conditioners, in terms of temperature control [22]. The selection and assessment of human body activity and light circumstances in the previous half hour are carried out among them in the switch type electrical model using the following equation:

$$
\lambda_{i}^{h}= \begin{cases}0, & \text { if } Z_{i}^{h} \Lambda_{i}^{h}<\gamma, \\ 1, & \text { if } Z_{i}^{h} \Lambda_{i}^{h}>\gamma .\end{cases}
$$

In (3), $\gamma$ and $\lambda_{i}^{h}$, respectively, represent the threshold value and the electrical switch status at time $h$. The comfort parameter $t_{k}$ of the switch appliance is calculated using the following equation:

$$
t_{k}=\sum_{i=1}^{m} \sum_{h=0}^{H} 0.5 \lambda_{i}^{h}
$$

where $m$ represents the number of switching appliances, and the user's comfort will be reduced due to the switching of appliances, and the number of switching appliances and comfort is in inverse proportion.

The comfort index is introduced to control the temperature control electrical appliances. The following equation expresses the comfort function: 


$$
\phi_{i}^{h}=\left\{\begin{array}{l}
W_{i}^{h}-W_{\text {in }}^{h}-\omega_{i}\left(W_{\text {out }}^{h}-W_{\text {in }}^{h}\right) \\
\text { if } W_{\text {on }}^{h}<W_{i}^{h}-\Delta W_{2} \\
0, \text { if } W_{i}^{h}-\Delta W \leq W_{\text {in }}^{h} \leq W_{i}^{h}+\Delta W_{1}, \\
W_{\text {in }}^{h}-W_{i}^{h}-\omega_{i}\left(W_{\text {out }}^{h}-W_{\text {in }}^{h}\right) \\
\text { if } W_{\text {in }}^{h}>W_{i}^{h}+\Delta W_{1}
\end{array}\right.
$$

where $W_{i}^{h}$ and $W_{i n}^{h}$ represent the set temperature of the electrical appliance and the indoor temperature, respectively, $W_{\text {out }}^{h}$ represents the outdoor temperature, and $\Delta W_{1}$ and $\Delta W_{2}$ represent the upper limit temperature and lower limit temperature of human body comfort, respectively. When optimizing comfort, you can also use the human body sensor to judge and set the temperature control appliance. The time range is set to $30 \mathrm{~min}$. If no human activity is detected in this interval, you can set it off. The following equation represents the functional expression:

$$
x_{i}^{h}=\left\{\begin{array}{ll}
0, & \text { if } x_{i}^{h}<\sigma \\
1, & \text { if } x_{i}^{h}>\sigma
\end{array},\right.
$$

where $x_{i}^{h}$ and $\sigma$, respectively, represent the threshold value of the electrical switch status and the human activity status. Use the following equation to express the comfort parameter $t_{c}$ of temperature control appliances:

$$
t_{c}=\sum_{i=1}^{n-m} \sum_{h=0}^{H} 0.5 x_{i}^{h} \phi_{i}^{h} .
$$

Use thefollowing equation to express the overall multiparameter comfort model:

$$
t_{k}+t_{c}=\sum_{i=1}^{n-m} \sum_{h=0}^{H} 0.5 x_{i}^{h} \lambda_{i}^{h}+\sum_{i=1}^{n-m} \sum_{h=0}^{H} 0.5 x_{i}^{h} \phi_{i}^{h} .
$$

(3) Load Control Based on PSO Algorithm. The advantages of PSO methods include good optimization performance, quick convergence, and reliance on fewer empirical factors. When solving models that demand a high level of precision, it offers a distinct advantage [23]. It is utilised to solve a multiobjective model for comfort and electricity prices in order to find the best load control strategy. The following is the model solution process carried out by the particle swarm optimization algorithm.

Suppose there are $m$ particles in a single particle group, $a_{i}=\left(a_{i 1}, a_{i 2}, \cdots, a_{i j}, \cdots, a_{i J}\right)$ which represents the position of each particle in the $j$-dimensional space, where $i=1,2, \cdots, m$ and $v_{i}=\left(v_{i 1}, v_{i 2}, \cdots, v_{i j}, \cdots, v_{i J}\right)$ represents the particle velocity. The following equation is used to update the position and velocity in the particle swarm optimization algorithm:

$$
\left\{\begin{array}{l}
a_{i, j}(t+1)=a_{i, j}(t)+v_{i, j}(t+1) \\
v_{i, j}(t+1)=\omega v_{i, j}(t)+c_{1} r_{1}\left[q_{i, j}-a_{i, j}(t)\right], \\
+c_{2} r_{2}\left[q_{g, j}-a_{i, j}(t)\right]
\end{array}\right.
$$

where $a_{i, j}(t)$ and $x_{i, j}(t+1)$, respectively, represent the velocity component and position component of the $t^{\text {th }}$ particle in the $j$-dimension. At the same time, they are related to the change in the input power of each node in the previous iterations during load control and the change in the power system after the $t^{\text {th }}$ iteration. $\omega$ and $q_{i, j}$, respectively, represent the inertia weight factor and the optimal position of the $i^{\text {th }}$ particle in the $j^{\text {th }}$ component. $c_{1}, c_{2}$ and $r_{1}, r_{2}$, respectively, represent the positive acceleration constant and the uniform random number in the interval of $[1,2] . q_{g, j}$ represents the $j^{\text {th }}$ component of the optimal position of the particle swarm.

Equation (9) shows that the particle updates the direction and speed of movement during the whole movement process based on the group's optimal position and its own optimal position. If the optimal path calculated does not include the global optimal solution, then the global optimal solution cannot be searched by the particles, resulting in a situation where a local optimal solution converges prematurely. In order to prevent it from happening, the fitness distance is used to solve the problem prematurely compared to the improved particle swarm optimization algorithm as the basis. In comparison to the particle swarm algorithm, the optimized and improved particle swarm algorithm can update not only the speed based on the most favourable location of the particle, but also the finest arrangement of the group, and each particle can also update the adjacent particle with a higher fitness value. To carry out learning, the neighbouring particles to be learned must be particles located at and near particle $i$.

To achieve this objective, it is essential to search for the particle $q_{n, j}$ with the maximum fitness distance ratio. The following equation is the $j$-th dimension maximum fitness distance ratio of particle $i$ :

$$
q_{n, j}=\max \frac{E(m)-E(i)}{\left|a_{m, j}-a_{i, j}\right|},
$$

where $E(i)$ and $a_{i, j}$, respectively, represent the fitness value of the $i$-th particle and the $j$-dimensional position of the $i$-th particle. $E(m)$ and $a_{m, j}$, respectively, represent the fitness value of the $m^{\text {th }}$ particle and the $j$-dimensional position of the $m^{\text {th }}$ particle. $\left|a_{m, j}-a_{i, j}\right|$ represents the distance between two particles in the $j$ dimension.

When updating and improving the optimized particle swarm algorithm, three factors need to be considered when the velocity of the $i$ particle and the $j$ dimension of the particle are optimized:

(a) the historical most favourable location of the particle itself

(b) the best possible position of the particle swarm

(c) the optimal historical position of the optimal neighbour

Using the improved particle swarm optimization algorithm (IPSO), update the particle position, and change the particle speed as given in the following equation: 


$$
\begin{aligned}
v_{i, j}(t+1)= & \omega v_{i, j}(t)+c_{1} r_{1}\left[q_{i, j}-a_{i, j}(t)\right], \\
& +c_{2} r_{2}\left[q_{g, j}-a_{i, j}(t)\right]+c_{3} r_{3}\left[q_{n, j}-a_{i, j}(t)\right],
\end{aligned}
$$

where $c_{3}$ and $r_{3}$, respectively, represent the positive acceleration constant and a random number uniformly distributed in the interval of $[1,2]$.

\section{Optimal Design}

4.1. Optimal Design of Multiobjective Smart Home HumanComputer Interaction Load Control Algorithm. To carry out the multiobjective smart home human-computer interaction load control algorithm design, construct the smart home human-computer interaction load control object model, and to obtain the fuzzy state constraint parameters of the smart home human-computer interaction load control, the following equation is devised:

$$
S_{s}=G_{h}+F_{k}+Y_{e}
$$

where $G_{h}$ represents the method parameter of single-ended fusion control, $F_{k}$ represents the rated current fusion parameter model of the smart home human-computer interactive load control system, and $Y_{e}$ represents the adaptive fusion control variable of the smart home human-computer interactive load control, which is passed through the AC side. The feeder analysis shows the reliability fusion characteristic quantity of the control terminal as shown in the following equation:

$$
M_{v}=\frac{H(w)+S_{s}}{x_{k}}+Y_{e},
$$

where $x_{k}$ represents the characteristic parameter of smart home control and $H(w)$ represents the load fluctuation suppression model of smart home man-machine interactive load control. The output reliability state adjustment state function of smart home man-machine interactive load control is obtained as given in the following equation:

$$
y(u)=M_{v}+\left[T_{p}+J(\theta)\right]
$$

where $T_{p}$ represents the characteristic parameter of PID control and $J(\theta)$ represents the method parameter of load flow volatility constraint control. The device-level control and protection system is constructed. Under the submodules under different voltage loads, the reliability fusion model is obtained, and the normal distribution simulation is adopted. The method to obtain the reliability load distribution parameters of the smart home man-machine interactive load control is given by the following equation:

$$
D_{m}(n)=\frac{R_{m}(o)+L_{n}}{k(n)}+\sum_{u=1} y(u) .
$$

Taking into account (15) lossless power suppression, the normal distribution fitting parameter analysis model of the smart home man-machine interactive load control system is constructed as $k(n)$, and the joint distribution relationship weight parameters of the smart home man-machine interactive load control meet $R_{m}(o)=R_{0}(o), L_{n}=1$. In the current load test subsystem of the control system, the parameter optimized and integrated output of the intelligent control system is shown in the following equation:

$$
M_{l}=Q_{o}+D_{m}(n)+K(n)
$$

According to (16) fusion of multidimensional sensor nodes, the joint fitness characteristic quantity $Q_{o}$ of smart home human-computer interaction load control is obtained, and the control sequence output is obtained as shown in the following equation:

$$
P_{k}=B_{h}+\left[R^{(0)}(m+n)+M_{l}\right]
$$

where $B_{h}$ represents the parameters of the smart home manmachine interactive load control system. The embedded control of the smart home man-machine interactive load control system is realised through the registers of the built-in module, and the predicted value of the reliability control is $R^{(0)}(m+n)$. At the flexible multistate switch node, the steady-state control equation is obtained as given by the following equation:

$$
B(w)=P_{k}+\frac{L(n)+P_{k}}{x_{n}} .
$$

In (18), $x_{n}$ represents the steady-state characteristic control function, $L(n)$ represents the method parameters of the support vector machine learning, and the frequencyduration parameter tracking learning model for constructing the smart home human-computer interaction load control system is given by the following equation :

$$
Z_{i}=\int_{w=1} B(w)+\Delta \lambda+R(s)
$$

In (19), $\Delta \lambda$ represents the frequency parameter of the control system, and $R(s)$ represents the full probability condition, instead of seeking the value of the derivative function, to realise the optimization design of the multiobjective smart home human-computer interaction load control algorithm.

\section{Experimental Outcome and Analysis}

5.1. Experimental Outcome. For experimental purpose, a house is taken as the research object having area of 109 square meters. It integrates the full domestic smart home equipment, including smart lighting systems and smart electrical control, using integrated wiring technology, security technology, automated control technology, network communication technology, and audio and video technology. Digital audio and video control, as well as security system control, contains information interaction features such as network connectivity, equipment automation, and home appliance information, in addition to conventional household functions. MATLAB simulator is used to build a simulation environment. Sensor equipment is used to collect the residential intelligence home-related data as input to carry out load control experiments. We have used the applicability method of hybrid broadcast broadband TV 
(AMHBBT) [5] and the smart home status and challenges associated with context-aware Internet of Things (SHS-IOT) [6] as the comparison methods. The experimental findings achieved using these two methods to run experiments on the simulation platform at the same time are in contrast to the method suggested in this paper.

To verify the convergence of the method, the numbers of particles and neighbours are set to 72 and 40 , the dimension is set to 4 , and the maximum inertia weighting factor and the minimum inertia weighting factor are 1.0 and 0.5 , respectively.

It can be observed from Figure 1 that the proposed method converges quickly, and it only needs 20 iterations to achieve a good fitness value. It has shown a stable state after 20 iterations, indicating that the proposed method has high applicability. The optimization result on benchmark is shown in Table 1; the rankings show that changing the function and dimension has minimal effect on the proposed method.

Table 1 depicts the results of comparative analysis of all the three methods used to control the load of the research object. The results of the change in electricity price of the research object within one year after statistical control are shown in Table 1.

Table 1 shows that when the two compared approaches are used to manage the load of the research object's smart house, the electricity price is greater, and the aim of the lowest electricity price is not realised. The outcome of the electricity price reduction attained using the proposed approach is bigger, indicating that the method in this paper regulates the load in the study region well. In the given table, the consumption of electricity is shown unitwise. The first method is not using any controlling mechanism and by using this method, more electricity is consumed, and the other three methods are using smart techniques including the proposed method which shows reduction in consumption of electricity each month. The proposed method is showing minimal consumption of energy.

Figure 2 shows the results of comparing the three methods within 24 hours of multiparameter comfort values.

It is analysed that the usage of smart electrical switches will diminish user comfort while using electrical switches instead of human-computer interface load control of smart homes. The more the switches that are employed are, the less comfortable the users will be. Therefore, in this study, the multiparameter comfort of smart homes is analysed. If minimal electric switches are used, the load control effect will be better. Figure 2 shows that, when compared to the other two methods, the multiparameter comfort values obtained after controlling the load are both higher, indicating good control. It is observed that the multiparameter comfort value of the study area is always less than 10. From this, it can be seen that, after using the method proposed in this paper to control the load of the smart home in the study area, a good comfort effect and control effect are obtained.

Table 2 shows the comparison of the three methods at different times. It reflects the changes in the power load of the research object.

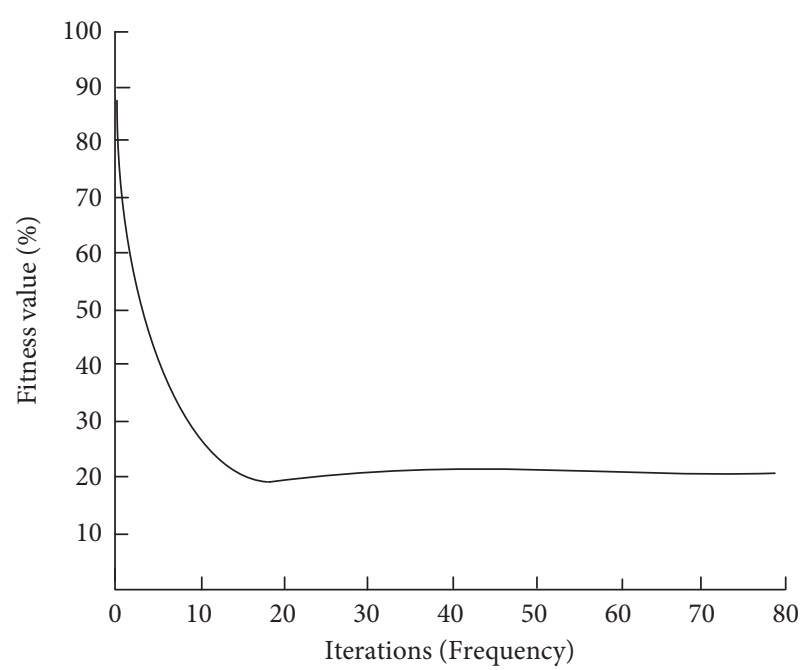

FIGURE 1: The convergence of the method in this paper.

Table 2 shows that the power load of the research object under the technique in this paper is always the lowest at different time periods, indicating that the approach in this paper can effectively regulate the power load of the research object. Statistics about the power consumption of the research object after using three methods to control the power load of the research object during one month are shown in Table 3.

It can be seen from Table 3 that after the two comparative methods are used to control the load of the research object, although the power consumption has been reduced, the reduction is not obvious, and the purpose of saving power has not been achieved. The power consumption of the object is significantly reduced, and the load control effect is better for the proposed method.

The average power reduction percentage of the three methods is calculated, and the results are shown in Figure 3.

Figure 3 shows that using proposed method to control the required area load can reduce electricity consumption by more than $40 \%$, indicating that using this method to control the required area load can save electricity and improve the economic benefits of smart home human-computer interaction load control.

One more study is conducted quarterly to check out the electricity consumption.

The analysis of consumption of electricity in four quarters is conducted. Some differences are obvious due to weather conditions as in extremely hot season and extremely cold season, the electricity consumption is more. The ordinary method or conventional method with other two stateof-the-art methods is taken up for comparative study and the performance of the algorithms is measured with our proposed approach. Table 4 shows that electricity consumption can be reduced by employing our proposed method up to a great extent.

5.2. Analysis. The current research is important for the development of load control issues in industry 4.0, smart cities, and smart homes. The case study is taken for smart 
TABLE 1: Comparison of electricity price results (RMB).

\begin{tabular}{lcccc}
\hline Month & No control method used & AMHBBT & SHS-IOT & Proposed method \\
\hline January & 112 & 106 & 114 & 98 \\
February & 105 & 95 & 97 & 87 \\
March & 101 & 92 & 110 & 83 \\
April & 116 & 104 & 97 & 99 \\
May & 105 & 96 & 127 & 85 \\
June & 138 & 115 & 128 & 94 \\
July & 146 & 137 & 114 & 101 \\
August & 139 & 120 & 101 & 96 \\
September & 118 & 103 & 99 & 89 \\
October & 109 & 95 & 94 & 85 \\
November & 96 & 87 & 92 & 72 \\
December & 95 & 81 & & 75 \\
\hline
\end{tabular}

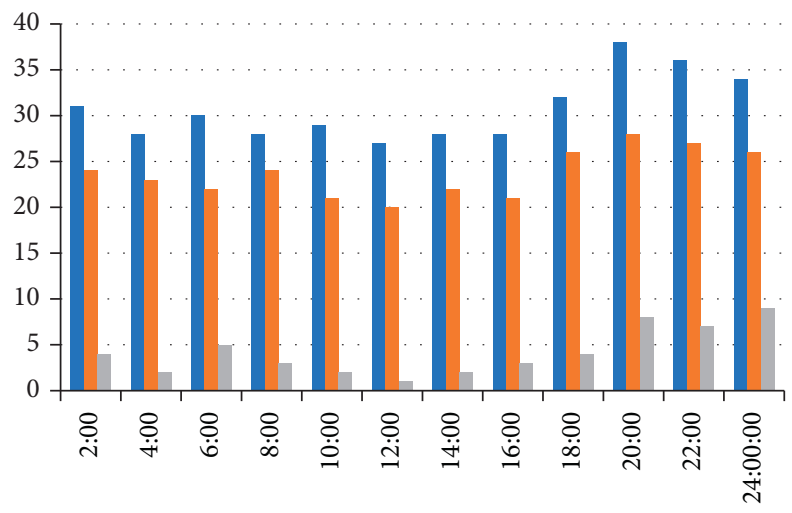

Time

$$
\begin{aligned}
& \text { AMHBBT } \\
& \text { SHS-IOT } \\
& \text { Proposed Method }
\end{aligned}
$$

FIGURE 2: Comparison results of multiparameter comfort values.

TABle 2: Comparison of power load changes (W).

\begin{tabular}{lccc}
\hline Time & AMHBBT & SHS-IOT & Proposed method \\
\hline $2: 00$ & 3541 & 3395 & 3157 \\
$4: 00$ & 3152 & 3054 & 2847 \\
$6: 00$ & 2984 & 2762 & 2605 \\
$8: 00$ & 2872 & 2762 & 2546 \\
$10: 00$ & 3851 & 3641 & 3541 \\
$12: 00$ & 3976 & 3854 & 3782 \\
$14: 00$ & 4105 & 3964 & 3895 \\
$16: 00$ & 4358 & 4251 & 4072 \\
$18: 00$ & 4596 & 4395 & 4257 \\
$20: 00$ & 4768 & 4384 & 4438 \\
$22: 00$ & 4927 & 4816 & 4689 \\
$24: 00$ & 4013 & 3841 & 3574 \\
\hline
\end{tabular}

home in the paper and the research work can be extended for smart cities or for industry 4.0. The novelty is in the proposed method, that is, handling multiple objectives for optimizing energy usage, for cutting down the usage cost, and for improving the electricity load in the smart home for optimal usage of energy. The limitations of the study are that
Table 3: Power consumption statistics $\left({ }^{\circ}\right)$.

\begin{tabular}{lcccc}
\hline Date & No control method used & AMHBBT & $\begin{array}{c}\text { SHS- } \\
\text { IOT }\end{array}$ & $\begin{array}{c}\text { Proposed } \\
\text { method }\end{array}$ \\
\hline 3 & 36.95 & 30.82 & 28.48 & 14.32 \\
6 & 38.24 & 33.41 & 27.65 & 10.64 \\
9 & 35.19 & 29.80 & 24.61 & 11.62 \\
12 & 37.02 & 32.94 & 25.34 & 9.52 \\
15 & 34.25 & 30.72 & 23.83 & 8.64 \\
18 & 31.87 & 28.65 & 22.41 & 7.65 \\
21 & 39.40 & 35.28 & 24.68 & 8.64 \\
24 & 37.62 & 32.84 & 25.72 & 10.66 \\
27 & 33.76 & 28.41 & 22.61 & 9.82 \\
30 & 34.08 & 30.58 & 21.34 & 7.68 \\
\hline
\end{tabular}

the proposed model is not tested for bigger houses, buildings, smart cities, and industries. The results prove that the proposed method is devised to control the required area load and can reduce electricity consumption by more than $40 \%$, indicating that using this method to control the required area load can save electricity and improve the economic benefits of smart home human-computer interaction load 


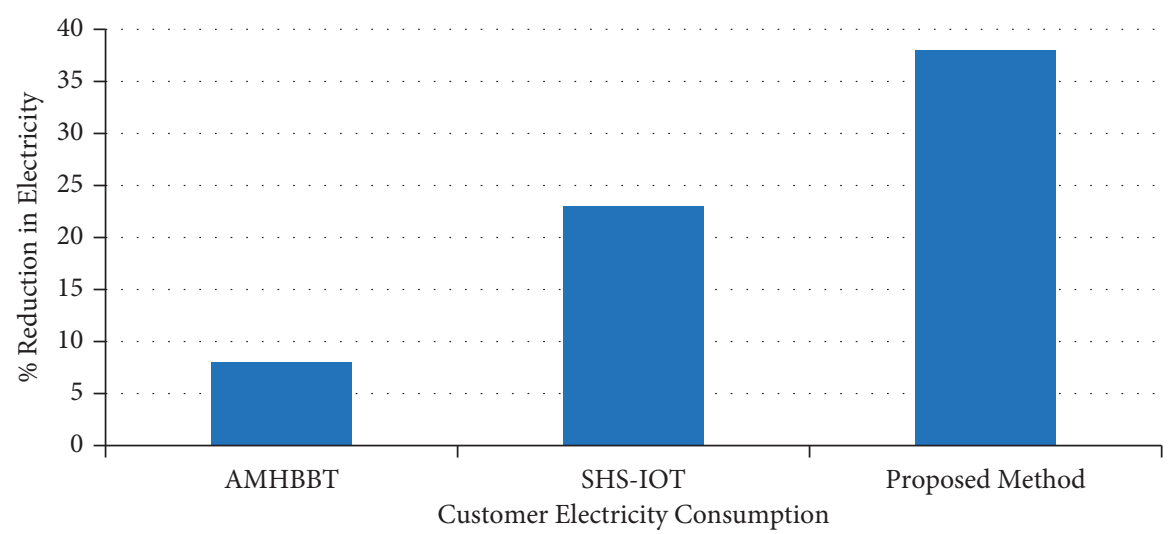

FIGURE 3: Results of the percentage reduction in power consumption.

TABLE 4: Comparison of quarterly consumption of electricity in Kilowatts.

\begin{tabular}{lcccc}
\hline Quarters & No control method used & AMHBBT & SHS-IOT & Proposed method \\
\hline First & 302 & 295 & 300 & 280 \\
Second & 346 & 315 & 320 & 281 \\
Third & 372 & 360 & 345 & 290 \\
Fourth & 300 & 278 & 285 & 252 \\
\hline
\end{tabular}

control. The proposed work constructs the comfort and electricity price multiobjective model and uses the fitness distance ratio as the basis to improve the particle swarm optimization algorithm for handling multiple objectives without considering the constraints. The model is compared with AMHBBT and SHS-IOT and the results of the proposed model are optimal, which realises the usage of multiobjective-based model for improving the smart home human-computer interaction. The proposed work also controls energy load, saves electricity costs and power consumption, and reduces the electricity load, ensuring that the residence realises the smart home human-computer interaction.

\section{Conclusion and Future Scope}

This paper presents multiobjective smart home humancomputer interaction load control algorithm, which combines the electricity price model and the multiparameter comfort model for the human-machine exchange. It constructs the comfort and electricity price multiobjective model and uses the fitness distance ratio as the basis to improve the particle swarm optimization algorithm. The model is compared with AMHBBT and SHS-IOT and the results of the proposed model are optimal, which realises multiobjective smart home human-computer interaction load control and saves electricity costs and power consumption. It also reduces the electricity load, ensuring that the residence realises the smart home human-computer interaction. Also at the same time, the electricity load is minimised. The results show that using the proposed method to control the required area load can reduce electricity consumption by more than $40 \%$, indicating that using this method to control the required area load can save electricity and improve the economic benefits of smart home human-computer interaction load control. The next plan in this direction is to study the three-dimensional emotional state model for multiobjective smart home human-computer interaction load control [24].

\section{Data Availability}

Data are sharable on request from the corresponding author.

\section{Conflicts of Interest}

The authors declare no conflicts of interest.

\section{Acknowledgments}

The authors are thankful to all who have supported them in completion of this research work.

\section{References}

[1] W. Moyle, J. Murfield, and K. Lion, "The effectiveness of smart home technologies to support the health outcomes of community-dwelling older adults living with dementia: a scoping review," International Journal of Medical Informatics, vol. 153, no. 9, Article ID 104513, 2021.

[2] M. Kaur, G. Kaur, P. K. Sharma, A. Jolfaei, and D. Singh, "Binary cuckoo search metaheuristic-based supercomputing framework for human behavior analysis in smart home," The Journal of Supercomputing, vol. 76, no. 15, pp. 2479-2502, 2020.

[3] L. Jiang, S. R. Sakhare, and M. Kaur, "Impact of industrial 4.0 on environment along with correlation between economic growth and carbon emissions," Int J Syst Assur EngManagement, 2021.

[4] M. Kaur and S. Kadam, "A novel multi-objective bacteria foraging optimization algorithm (MOBFOA) for multi-objective scheduling," Applied Soft Computing, vol. 66, pp. 183-195, 2018. 
[5] O. Taiwo and A. E. Ezugwu, "Internet of things-based intelligent smart home control system," Security and Communication Networks, vol. 2021, Article ID 9928254, 17 pages, 2021.

[6] M. Manic, D. Wijayasekara, K. Amarasinghe, and J. J. Rodriguez-Andina, "Building energy management systems: the age of intelligent and adaptive buildings," IEEE Industrial Electronics Magazine, vol. 10, no. 1, pp. 25-39, 2016.

[7] G. Paravati and V. Gatteschi, "Human-computer interaction in smart environments," Sensors, vol. 15, no. 8, pp. 19487-19494, 2015.

[8] Z. Ali-Khan, A. Khalid, N. Javaid, A. Haseeb, T. Saba, and M. Shafiq, "Exploiting nature-inspired-based artificial intelligence techniques for coordinated day-ahead scheduling to efficiently manage energy in smart grid," IEEE Access, vol. 7, 2019.

[9] F. Ren and Y. Bao, "A review on human-computer interaction and intelligent robots," International Journal of Information Technology and Decision Making, vol. 19, no. 1, pp. 05-47, 2020.

[10] X. Huang, V. Jagota, E. Espinoza-Muñoz, and J. FloresAlbornoz, "Tourist hot spots prediction model based on optimized neural network algorithm," International Journal of System Assurance Engineering and Management, 2021.

[11] M. V. Gladence, M. V. Anu, R. Rathna, and E. Brumancia, "Recommender system for home automation using IoT and artificial intelligence," Journal of Ambient Intelligence and Humanized Computing, pp. 1-9, 2020.

[12] A. Chatterjee, S. Paul, and B. Ganguly, "Real time multiobjective energy management of a smart home," in Proceedings of the 2020 IEEE International Conference on Power Electronics, Drives and Energy Systems (PEDES), Jaipur, India, December 2020.

[13] J. Bhola, M. Shabaz, G. Dhiman, S. Vimal, P. Subbulakshmi, and S. K. Soni, "Performance evaluation of multilayer clustering network using distributed energy efficient clustering with enhanced threshold protocol," Wireless Personal Communications, 2021.

[14] M. Kaur, "Elitist multi-objective bacterial foraging evolutionary algorithm for multi-criteria based grid scheduling problem," in Proceedings of the 2016 International Conference on Internet of Things and Applications (IOTA), pp. 431-436, Pune, India, January 2016.

[15] R. Zhang, S He, X. Yang et al., "An EOG-based humanmachine interface to control a smart home environment for patients with severe spinal cord injuries," IEEE Transactions on Biomedical Engineering, vol. 66, no. 1, pp. 89-100, 2019.

[16] N. G. Paterakis, O. Erdinc, I. N. Pappi, A. G. Bakirtzis, and J. P. S. Catalao, "Coordinated operation of a $n$ of smart households comprising electric vehicles, energy storage and distributed generation," IEEE Transactions on Smart Grid, vol. 7, no. 6, pp. 2736-2747, 2016.

[17] M. Kaur, "Multi-objective evolution-based scheduling of computational intensive applications in grid environment," in Proceedings of the Proc. of the Int. Conf. on Data Engg and Comm. Tech. Adv. in Int. Sys. \& Comp, Springer, Singapore, Auguest 2016.

[18] I. Machorro-Cano, G. Alor-Hernandez, M. A. ParedesValverde, L. Rodriguez-Mazahua, J. L. Sanchez-Cervantes, and J. O. Olmedo-Aguirre, "Hems-IoT: A big data and machine learning-based smart home system for energy saving," Energies, vol. 13, 2020.

[19] D. Kundu, M. E. Khallil, T. K. Das, A. A. Mamun, and A. Musha, "Smart home automation system using on IoT,"
International Journal of Scientific Engineering and Research, vol. 11, no. 6, pp. 697-701, 2020.

[20] L.-D. Liao, C.-C. Chuang, T.-R. Ger et al., "Design and validation of a multifunctional android-based smart home control and monitoring system," IEEE Access, vol. 7, pp. 163313-163322, 2019.

[21] C. Gavrila, V. Popescu, M. Fadda, M. Anedda, and M. Murroni, "On the suitability of HbbTV for unified smart home experience," IEEE Transactions on Broadcasting, vol. 67, no. 1, pp. 1-10, 2020.

[22] R. Majeed, N. A. Abdullah, I. Ashraf, Y B Zikria, M F Mushtaq, and M Umer, "An intelligent, secure, and smart home automation system," Scientific Programming, vol. 2020, Article ID 4579291, 14 pages, 2020.

[23] O. Taiwo, A. E. Ezugwu, N. Rana, and S. i. M. Abdulhamid, "Smart home automation system using ZigBee, Bluetooth and Arduino technologies," Computational Science and Its Applications - ICCSA 2020, vol. 12254, pp. 587-597, 2020.

[24] Z. A. Almusaylim and N. Zaman, "A review on smart home present state and challenges: linked to context-awareness internet of things (IoT)," Wireless Networks, vol. 25, no. 6, pp. 3193-3204, 2019. 